\title{
Significance of vomiting after head injury
}

\author{
P A Nee, J M Hadfield, D W Yates, E B Faragher
}

\begin{abstract}
Objectives-To determine whether the presence and severity of post-traumatic vomiting can predict the risk of a skull vault fracture in adults and children.

Methods-Data were analysed relating to a consecutive series of 5416 patients including children who presented to an emergency service in the United Kingdom during a 1 year study period with a principal diagnosis of head injury. Characteristics studied were age, sex, speed of impact, level of consciousness on arrival, incidence of skull fracture, and the presence and severity of post-traumatic vomiting.

Results-The overall incidence of posttraumatic vomiting was $7 \%$ in adults and $12 \%$ in children. In patients with a skull fracture the incidence of post-traumatic vomiting was $28 \%$ in adults and $33 \%$ in children. Post-traumatic vomiting was associated with a fourfold increase in the relative risk for a skull fracture. Nausea alone did not increase the risk of a skull fracture and multiple episodes of vomiting were no more significant than a single episode. In patients who were fully alert at presentation, post-traumatic vomiting was associated with a twofold increase in relative risk for a skull fracture.
\end{abstract}

Conclusion-These results support the incorporation of enquiry about vomiting into the guidelines for skull radiography.

Accident and

Emergency

Department, Whiston

Hospital, Warrington

Road, Merseyside, UK

P A Nee

Department of Emergency Medicine, Hope Hospital, Stott Lane, Salford, UK

J $M$ Hadfield

D W Yates

Medical Statistics Research Support Unit, University of Manchester Medical School, Oxford Road, Manchester, UK

E B Faragher

Correspondence to:

Mrs J M Hadfield,

Department of Emergency

Medicine, Hope Hospital,

Stott Lane, Salford M6

8HD, UK. email

jhadfield@gen.srht.nwest.

nhs.uk

Received 8 August 1997 and in revised form

23 October 1998

Accepted 30 October 1998
One episode of vomiting seems to be as significant as multiple episodes.

(F Neurol Neurosurg Psychiatry 1999;66:470-473)

Keywords: head injuries, vomiting, skull radiography, skull fracture

The average emergency department in the United Kingdom receives about 5000 patients with a head injury each year. More than eight out of 10 will be classified as mild, scoring 13 or more points on the Glasgow coma scale on first assessment. ${ }^{1}$ In this population it is well established that the presence of a skull fracture is associated with a 200 -fold increase for the risk of development of an extradural haematoma, a condition with an outcome that is significantly improved by early detection and treatment. $^{2}$

The preferred investigation for the detection of a haematoma is CT. In the United Kingdom this is usually reserved for patients with a depressed level of consciousness, focal neurological signs, or a skull fracture. Thus skull radiography remains the principal triage tool for determining which patients should be admitted to hospital for observation or referred for CT examination. ${ }^{3}$ Not all patients with mild head injury have a skull radiograph. Patient selection is based on guidelines, written by a group of neurosurgeons, and available in most United Kingdom emergency departments. ${ }^{4}$

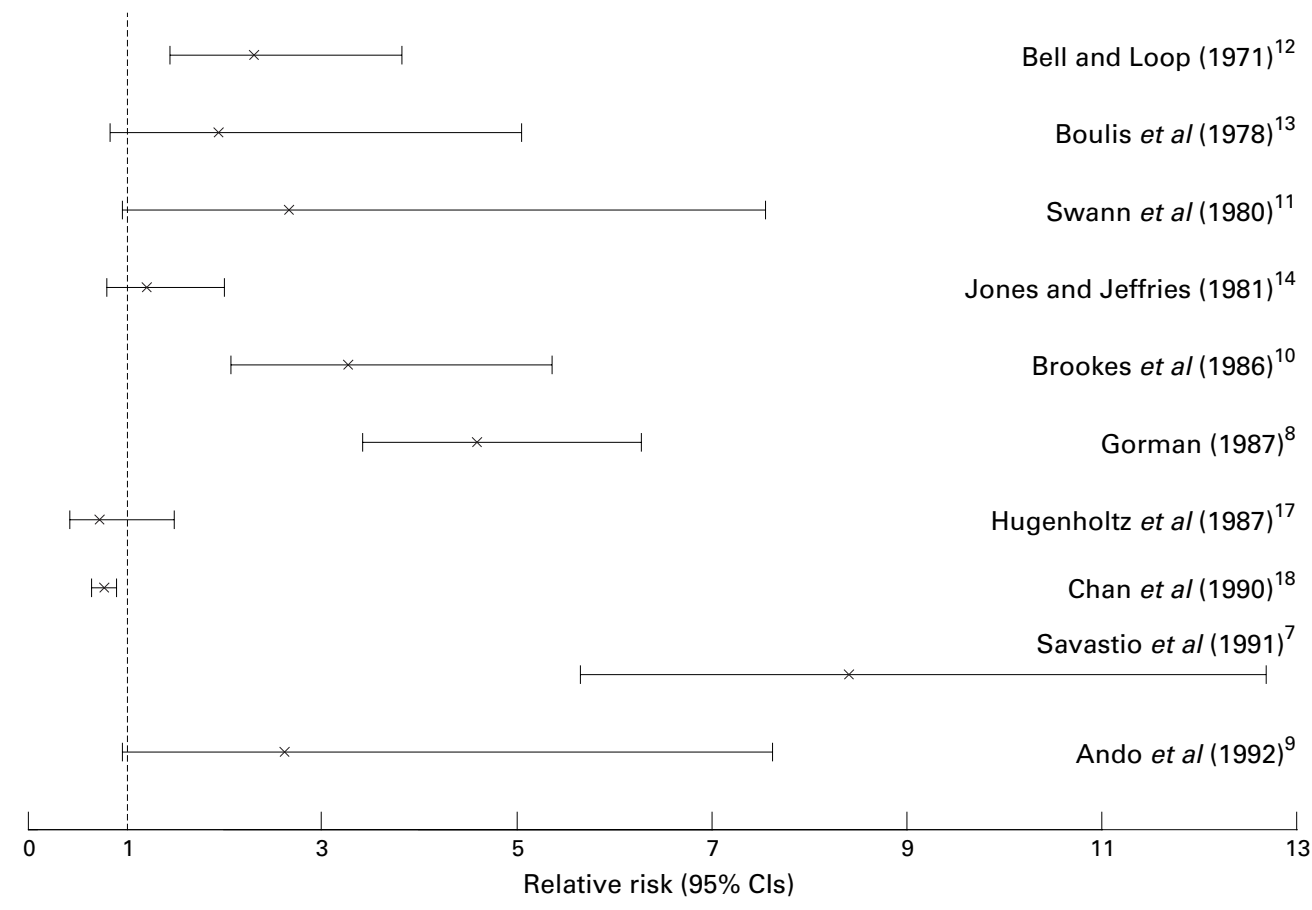

Skull fracture in the presence of nausea or vomiting: published research. 
Table 1 Incidence (\%) of skull fracture relative to severity of post-traumatic vomiting $(P T V)+$

\begin{tabular}{lllll}
\hline Symptoms of PTV & Nil & Nausea only & Single vomit & Multiple vomits \\
\hline Adults & $2359(86.0)$ & $200(7.3)$ & $149(5.4)$ & $35(1.3)$ \\
$\begin{array}{l}\text { No fracture } \\
\text { Fracture }\end{array}$ & $34(68.0)$ & $2(4.0)$ & $12(24.0)$ & $2(4.0)$ \\
Children & $2088(82.7)$ & $140(5.5)$ & $224(8.9)$ & $73(2.9)$ \\
No fracture & $31(64.6)$ & $1(2.1)$ & $12(25.0)$ & $4(8.3)$ \\
Fracture & &
\end{tabular}

†Severity of PTV not recorded for 42 adults and eight children.

Table 2 Relative risk of skull fracture in relation to presenting characteristics

\begin{tabular}{lll}
\hline Presenting characteristic & Relative risk & $(95 \%$ CI $)$ \\
\hline PTV: & 1 & \\
$\quad$ Nil & 0.75 & $(0.27-2.11)$ \\
$\quad$ Nausea & 4.28 & $(2.76-6.63)$ \\
$\quad$ Vomiting & 1 & \\
Age group: & 1.02 & $(0.68-1.51)$ \\
$\quad$ Adults & & \\
$\quad$ Children & 1 & \\
Sex: & 0.89 & $(0.58-1.37)$ \\
$\quad$ Female & & \\
$\quad$ Male & 1 & \\
Consciousness level: & 7.50 & $(5.02-11.2)$ \\
$\quad$ alert & & \\
altered & 1 & $(3.62-20.3)$ \\
Speed of impact: & 8.58 & $(16.1-92.8)$ \\
$\quad$ Low & 38.7 & \\
$\quad$ Medium & High &
\end{tabular}

Although evidence based, they are neither entirely sensitive nor specific.

Post-traumatic vomiting has been reported as a feature of missed intracranial haematoma ${ }^{56}$ but there is no clear evidence that it is an independent risk factor. ${ }^{7-14}$ Some studies do regard vomiting as evidence of possible intracranial injury and link it with headache as an indication for admission and extended observation. ${ }^{15} 16$ Others have concluded that vomiting confers no additional risk in patients presenting with normal consciousness. ${ }^{17} 18$

The purpose of the present investigation was to determine whether the presence and severity of post-traumatic vomiting can predict the risk of a skull vault fracture in adults and children. Demonstration of an adequate specificity and sensitivity to predict fracture in alert patients would indicate that an enquiry about vomiting should be incorporated into the guidelines for skull radiography after a head injury.

\section{Patients and methods}

A computer aided (Medline) search of the relevant literature published between 1986 and 1996 was undertaken. A hand search for similar studies published between 1970 and 1985 was also undertaken using Index Medicus. Studies were included for analysis when incidence rates for vomiting in patient groups with and without skull fracture were stated or could be calculated from the data provided. Relative risk with $95 \%$ confidence intervals ( $95 \%$ CIs) for skull fracture in the presence of vomiting were calculated for all studies with usable data published during the past 26 years.

An analysis was also undertaken of the presenting characteristics of a consecutive series of 5416 patients with head injury who attended the emergency department in Salford over a 1 year period. An assessment was made of age, sex, the speed of the impact categorised as high, medium, or low, level of consciousness on arrival, whether the patient had sustained any post-traumatic amnesia, the presence of nausea, the frequency and severity of any vomiting, and the presence of a skull fracture. Other data from this series have been reported previously. ${ }^{19}$

Follow up data on reattendances, complications, and deaths were obtained by reviewing emergency department records and admissions to neurosurgical and paediatric units serving the Salford district. The national records of the Registrar General on deaths relating to cranial trauma in Salford were examined for the period of the study and for a further 12 months. In this way as complete a record as possible was obtained on the outcome of all patients up to 1 year after presentation.

Fracture incidence rates were computed according to the presenting characteristics. Because of the small incidence of skull fractures, relative risks and their $95 \%$ CIs were estimated from odds ratios computed using standard multiple logistic regression methods. The presence or absence of a skull fracture was used as the dependent variable. Separate analyses were undertaken for adults and children. All computations were carried out using the SPSS/PC and CIA computer programs. ${ }^{20}$

Table 3 Relative risk of skull fracture with differing severity of post-traumatic vomiting (PVT)

\begin{tabular}{|c|c|c|c|c|}
\hline & \multicolumn{4}{|c|}{ Presenting characteristic } \\
\hline & Nil & Nausea only & Single vomit & Multiple vomits \\
\hline All patients combined & 1 & $0.78(0.28-2.21)$ & $4.31(2.65-7.02)$ & $3.72(1.55-8.92)$ \\
\hline \multicolumn{5}{|l|}{ Age group: } \\
\hline Adults & 1 & $0.69(0.16-3.00)$ & $5.59(2.80-11.2)$ & $3.96(0.89-17.7)$ \\
\hline Children & 1 & $0.48(0.06-3.70)$ & $3.61(1.80-7.23)$ & $3.69(1.24-11.0)$ \\
\hline \multicolumn{5}{|l|}{ Post-traumatic amnesia: } \\
\hline Present & 1 & $0.85(0.31-2.30)$ & $4.44(2.83-6.98)$ & $3.28(0.90-12.0)$ \\
\hline Absent & 1 & $1.08(0.39-3.02)$ & $3.31(1.85-5.92)$ & $2.34(0.62-8.83)$ \\
\hline \multicolumn{5}{|l|}{ Consciousness: } \\
\hline Alert & 1 & $0.64(0.15-2.73)$ & $2.24(0.93-5.39)$ & $3.15(0.72-13.7)$ \\
\hline Altered & 1 & $0.53(0.12-2.40)$ & $2.01(1.03-3.92)$ & $1.13(0.36-3.49)$ \\
\hline \multicolumn{5}{|l|}{ Speed of impact: } \\
\hline Low & 1 & $\dagger$ & $5.58(0.55-56.6)$ & $\dagger$ \\
\hline Medium & 1 & $0.53(0.12-2.27)$ & $2.00(0.91-4.42)$ & $1.49(0.34-6.48)$ \\
\hline High & 1 & $0.77(0.17-3.48)$ & $5.62(2.68-11.8)$ & $4.84(1.43-16.4)$ \\
\hline
\end{tabular}

†Too few skull fractures to estimate relative risk.

Values are relative risk $(95 \% \mathrm{CI})$. 


\section{Results}

The figure contains a summary of previously published studies relating post-traumatic vomiting with relative risk for a skull fracture. ${ }^{7-16}$

In the present study data were collected on 5416 patients of whom 2581 (48\%) were children under the age of 16 . One hundred and six (2\%) patients had a skull fracture; severity of post-traumatic vomiting was not recorded for eight of these patients. The overall incidence of vomiting was $7 \%$ in adults and $12 \%$ in children. Table 1 shows that in patients with a skull fracture the incidence of vomiting was $28 \%(95 \%$ CI $16 \%-48 \%)$ in adults and $33 \%$ $(20 \%-48 \%)$ in children. Sensitivity and specificity levels for vomiting as a predictor of skull fracture were $28.0 \%$ and $93.3 \%$ respectively for adults and $33.3 \%$ and $88.2 \%$ respectively for children.

Vomiting was associated with a roughly fourfold increase in the risk of skull fracture (table 2). Fracture rates were independent of sex and there was no difference between adults and children. Altered consciousness at presentation and high or medium velocity impacts were also associated with an increased relative risk for a skull fracture.

Table 3 shows the overall relation between skull fracture and the severity of vomiting and by presenting characteristics. Nausea alone did not increase the risk of a skull fracture and multiple episodes of vomiting were no more significant than a single episode. The correlation between vomiting and skull fracture was significant for both adults and children and for both sexes.

The presence of one or more episodes of vomiting increased the relative risk of a skull fracture irrespective of the presence or absence of post-traumatic amnesia, altered consciousness on arrival, or the speed of the impact.

\section{Discussion}

Earlier studies have shown wide variations in the estimation of an association between posttraumatic vomiting and skull fracture (figure). The most likely explanation for this is that different populations were being examined. Many reports are based on fairly small numbers of patients, ${ }^{9}{ }^{16}$ some do not distinguish between adults and children and others do not differentiate between different grades of severity of brain injury. Symptoms of nausea, vomiting, and headache are often not clearly distinguished.

We have shown that the occurrence of vomiting after brain injury is independently associated with an increased incidence of skull fracture in alert patients. There was no significant difference between adults and children. Although the incidence of vomiting was higher in children and the results support the popular contention that the threshold for posttraumatic vomiting is lower in children, vomiting did indicate a significantly increased risk of fracture. Multiple episodes of vomiting were no more predictive than a single episode although the $95 \%$ CIs were wide reflecting fewer patients in this group.
The value of post-traumatic vomiting as an independent risk factor can be assessed by considering the relative risk of skull fracture in alert patients. Among patients with a coma score in the range of 13-15 an episode of vomiting was associated with a greater than twofold increase in relative risk for a skull fracture. The 95\% CIs indicate a trend not quite reaching significance. Post-traumatic vomiting predicted the risk of a skull fracture irrespective of the level of consciousness, the speed of the impact, or the presence of post-traumatic amnesia.

The precise mechanism of post-traumatic vomiting is unknown but it is likely that contact forces (impact) are less important than inertial forces (impulse) in its aetiology. Whereas symptoms such as loss of consciousness and post-traumatic vomiting are induced by head motion, skull fracture depends on contact forces. In most injuries the two phenomena occur together. ${ }^{21}$ Forces sufficient to cause linear skull fracture will also set the head into motion causing shearing and compressive strain within the brain due to differential rates of acceleration of the different components of the brain and neuraxis. ${ }^{21}$

Brain deformations are greatest some distance from the site of impact and so shearing forces are maximal in the brain stem whenever the head moves in the sagittal plane..$^{22}$ This may lead to transient changes in the brain stem causing stimulation of the vomiting centre in the reticular formation of the lateral medulla. The exact pathways have yet to be identified but it is known that brain stem auditory evoked potentials are markedly different in children with mild brain injury with vomiting compared with those without. ${ }^{23}$

Post-traumatic vomiting was more common after high velocity impacts in which the conscious level was altered and when posttraumatic amnesia was present. The same inertial forces that induce vomiting may have been disrupting the neural connections between the cortex and subcortical region on which normal consciousness depends. Therefore posttraumatic vomiting may be regarded as testimony to the violence of the cranial trauma.

These results indicate that a question about vomiting in the guidelines for skull radiography would increase their sensitivity. This should lead to a reduction in the mortality and morbidity which can occur when definitive treatment for intracranial haematoma is delayed because of failure to identify a skull fracture and a consequent failure to admit the patient for observation.

1 Miller JD, Jones PA. The work of a regional head injury service. Lancet 1985;1:1141-4.

2 Mendelow AD, Teasdale G, Jennett B, et al. Risks of intracranial haematoma in head injured adults. BMF 1993;287 1173-6.

3 Teasdale GM, Murray G, Anderson E, et al. Risks of acute traumatic intracranial haematoma in children and adults: implications for managing head injuries. BMf 1990;300: 363-7.

4 Briggs $\mathrm{M}$, Clarke P, Crockard A, et al. Guidelines for initial management after head injury in adults. Suggestions from management after head injury in adults. Suggestion

5 Nee PA, Phillips BM, Bannister CM. Extradural haematoma in a child after an apparently mild head injury. BMF 1993;306:1665-6. 
6 Duus BR, Boesen T, Kruse KV, et al. Prognostic signs in the evaluation of patients with minor head injury. Br F Surg

7 Savastio G, Golfieri R, Pastore Trossello M, et al. Trauma cranico: predittivita dei sintomi di presentazione come screening per lo studio radiologico. Radiol Med (Torino) 1991;82:769-75

8 Gorman DF. The utility of post traumatic skull $x$ rays. Archives of Emergency Medicine 1987;4:141-50.

9 Ando S, Otani M, Moritake K. Clinical analysis of post -traumatic vomiting. Acta Neurochir Wein 1992;119:97-100.

10 Brookes M, Mendelow AD, MacMillan R, et al. Which head injured patients have a skull fracture? Archives of Emergency Medicine 1986;3:41-2.

11 Swann IJ, MacMillan R, Strong I. Head injuries in an inner city accident and emergency department. Injury 1980;12: 274-8.

12 Bell RS, Loop JW. The utility and futility of radiographic skull examination. $N$ Engl f Med 1971;284:236-9.

13 Buolis ZF, Dick R, Barnes NR. Head injuries in childrenaetiology, symptoms, physical findings, and $x$ ray wastage. aetiology, symptoms, physical
Br F Radiol 1978;51:851-4.

14 Jones JJ, Jeffries RV. Relative risk of alternative admission policies for patients with head injuries. Lancet 1981;1:850-3.

15 Masters SJ, McClean PM, Arcarese JS, et al. Skull x-ray examinations after head trauma. Recommendations by a multidisciplinary panel and validation study. $N$ Engl F Med 1987;316:84-91.
16 Lee ST, Liu TN, Wong YS, et al. Relative risk of deterioration after mild closed head injury. Acta Neurochir Wein 1995;135:136-40.

17 Hugenholtz H, Izukawa D, Shear $\mathrm{P}$, et al. Vomiting in children following head injury. Child's Nerv Syst 1987;3: 266-70.

18 Chan K-H, Yue CP, Mann KS, The risk of intracranial complications in paediatric head injury. Results of multivariate analysis. Childs Nerv Syst 1990;6:27-9.

19 Nee PA, Hadfield JM, Yates DW. Biomechanical factors in patient selection for skull radiography after head injury. Injury 1993;24:471-5.

20 Gardner MJ, Altman DG. Statistics with confidence and statistical guidelines. London: British Medical Association, 1989.

21 Gennarelli TA. The state of the art of head injury biomechanics. In: 29th Proceedings of the American Association for Automotive Medicine. Washington, DC:AAAM, ation for Auto $1985 ; 447-63$.

22 Aldman B. An analytical approach to impact biomechanics of head and neck injury In: 30th Proceedings of the American Association for Automotive Medicine. Montreal, Quebec: AAAM, 1986.

23 Hashimoto I, Nemoto S, Sano K. Hyperexcitable state of the brainstem in children with post-traumatic vomiting as evidenced by brainstem auditory evoked potentials. Neurol Res 1984;6:81-4. 